\title{
MiR-186 bidirectionally regulates cisplatin sensitivity of ovarian cancer cells via suppressing targets PIK3R3 and PTEN and upregulating APAF1 expression
}

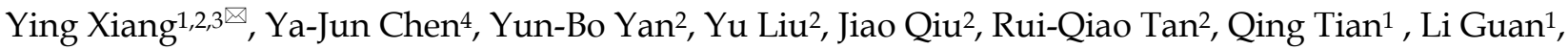 \\ Shuai-Shuai Niu ${ }^{1}$, Hong-Wu Xin ${ }^{2,5}{ }^{\square}$ \\ 1. Department of Cell Biology and Genetics, School of Basic Medicine, Health Science Center, Yangtze University, Jingzhou, Hubei 434023, China. \\ 2. Laboratory of Oncology, Center for Molecular Medicine, School of Basic Medicine, Health Science Center, Yangtze University, 1 Nanhuan Road, Jingzhou, \\ Hubei 434023, China \\ 3. The First School of Clinical Medicine, Health Science Center, Yangtze University, Nanhuan Road, Jingzhou, Hubei 434023, China. \\ 4. Department of Oncology, Jingzhou Central Hospital, The Second Clinical Medical College, Yangtze University, Jingzhou, Hubei 434023, China. \\ 5. Department of Molecular Biology and Biochemistry, School of Basic Medicine, Health Science Center, Yangtze University, Jingzhou, Hubei 434023, China.
}

$\triangle$ Corresponding authors: Ying Xiang, PhD, Department of Cell Biology and Genetics, School of Basic Medicine, Health Science Center, Yangtze University, Jingzhou, Hubei 434023, China. Phone: +86 15107212530; Fax: 0716-8062633; Email: xying316@163.com. Or Hong-Wu Xin, PhD, MD,Laboratory of Oncology, Center for Molecular Medicine, School of Basic Medicine, Health Science Center, Yangtze University, 1 Nanhuan Road, Jingzhou, Hubei 434023, China. Phone: +86 13311055391; Fax: 0716-8062633; Email: hongwu_xin@126.com

(C) The author(s). This is an open access article distributed under the terms of the Creative Commons Attribution License (https://creativecommons.org/licenses/by/4.0/). See http://ivyspring.com/terms for full terms and conditions.

Received: 2019.10.13; Accepted: 2020.02.25; Published: 2020.03.13

\begin{abstract}
Ovarian cancer is a highly lethal malignancy in the female reproductive system. Platinum drugs, represented by cisplatin, are the first-line chemotherapeutic agents for treatment of various malignancies including ovarian cancer, but drug resistance leads to chemotherapy failure. MicroRNAs emerged as promising molecules in reversal of cisplatin resistance. MiR-186 was reported to be downregulated in the cisplatin-resistant ovarian cell lines and miR-186 expression increased cisplatin sensitivity. However, we found the bidirectional regulatory effects of miR-186 on cisplatin sensitivity for the first time that overexpression of miR-186 at low concentration increased the cisplatin sensitivity of ovarian cancer cells A2780/DDP, while high concentration of miR-186 decreased the cisplatin sensitivity. The survival assay in other types of cancer cell lines verified the bidirectional regulatory function of miR-186 on cisplatin sensitivity in dose and cell type dependent manners. MiR-186 suppressed the protein levels of PTEN and PIK3R3 dose-dependently, which are opposite regulatory molecules of the oncogenic AKT pathway. MiR-186 also enhanced the protein levels of apoptotic gene APAFI dose-dependently. We proposed the final effects of PTEN and APAF1 outweighed PIK3R3 when miR-186 at low concentration so as to increase the cisplatin sensitivity of ovarian cancer cells, while the final effects of PIK3R3 outweighed PTEN and APAFI when miR-186 at high concentration so as to decrease the cisplatin sensitivity. We concluded the outcome of regulation of these opposite functional molecules contributed to the bidirectional regulatory effects of miR-186 in ovarian cancer cisplatin sensitivity. It deserves more attentions when developing therapeutic strategies based on the bidirectional functional miRNAs.
\end{abstract}

Key words: ovarian cancer, miR-186, cisplatin sensitivity, dose-dependent, bidirectional regulation

\section{Introduction}

Ovarian cancer is the third most common malignancy and the second highly lethal malignancy in the female reproductive system, globally, according to the latest global cancer status data (GLOBCAN2018) [1]. Platinum drugs, represented by cisplatin, are the first-line chemotherapeutic agents for various malignancies including ovarian cancer [2].
Although the majority of patients with ovarian cancer can achieve complete remission at the initial stage with platinum-based chemotherapy, about $80 \%$ of patients show drug resistance at the later stage [3]. Drug resistance leads to the failure of chemotherapy as well as in other cancers. 
AKT is the most important effector of PI3K signaling. Class I PI3Ks phosphorylates PI4, 5P2 to PIP3, thus activating AKT. Phosphatase and tensin homolog (PTEN) dephosphorylates PIP3 to PI4, 5P2, negatively regulating AKT pathway. Activated AKT is able to promote cell survival, proliferation, and cause changes of metabolic pathways via its multiple downstream targets such as GSK3, FOXO and mTORC1 [4]. Inhibition of PI3K/AKT was reported to increase cisplatin sensitivity of cisplatin-resistant breast cancer cells [5]. Recently, the AKT pathway was confirmed to be activated in cisplatin resistant ovarian cancer [6].

MicroRNAs (miRNAs), non-coding RNAs with a length of 19-25nt, regulates gene expression post-transcriptionally via binding to the 3 '-untranslated regions (UTR) of target mRNAs [7]. MiRNAs are essential in physiological and pathological processes, including in regulating cisplatin sensitivity [8]. MiR-186 was documented as an tumor suppressor miRNA in the majority of studies, such as in carcinomas of prostate [9], breast [10] and liver [11], while some reports verified miR-186 as an oncomir $[12,13]$. We confirmed that miR-186 was downregulated in the cisplatin-resistant ovarian cell lines SKOV3/DDP and A2780/DDP cells, which were concordant to the previous reports [14, 15]. However, we firstly found the bidirectional regulatory effects of miR-186 on cisplatin sensitivity in ovarian cancer cells: low concentration of miR-186 increased the cisplatin sensitivity of ovarian cancer cells, while high concentration of miR-186 decreased the cisplatin sensitivity. The survival assay in other cancer cell lines revealed that miR-186 increased cisplatin sensitivity was dependent on its dose and cell types.

To investigate the mechanism of the bidirectional regulatory effects of miR-186 in ovarian cancer cisplatin sensitivity, multiple softwares of targets prediction were used to explore the potential targets. PTEN, PIK3R3 (phosphoinositide 3-kinase regulatory subunit 3) and APAF1 (apoptotic protease activating factor-1), were predicated as potential targets of miR-186. The dual reporter luciferase assay showed miR-186 inhibited all of these UTRs directly, and western blotting assay showed overexpression of miR-186 decreased the protein levels of PTEN and PIK3R3, which were two opposite functional molecules of AKT pathway, but increased the protein levels of apoptotic gene APAF1 in a dose dependent manner. We concluded the outcome of regulation of these opposite functional molecules contributed to the bidirectional regulatory effects of miR-186 in ovarian cancer cisplatin sensitivity.

\section{Materials and Methods}

\section{Cell culture and Transfection}

All human cell lines including cisplatin-resistant ovarian cancer cells SKOV3/DDP and A2780/DDP, and their parental cells SKOV3 and A2780, non-smallcell lung cancer cells A549, colon cancer cells HCT116, hepatic carcinoma cells HepG2, breast cancer cells SKBR3, and human embryonic kidney (HEK) 293T cells were conserved in our laboratory. The HCT116 cells in MycCoy's 5A medium (Senrui, China) and the other cells in Dulbecco's modified Eagle's medium (Invitrogen, USA) with 10\% fetal bovine serum and $1 \%$ penicillin/streptomycin, were cultured in a humid atmosphere containing $5 \% \quad \mathrm{CO} 2$ at $37{ }^{\circ} \mathrm{C}$. Lipofectamine 2000 (Invitrogen) was used for transfection with oligonucleotides miR-186 mimic or NC (Gene Pharma Company, Shanghai, China). The $\mathrm{NC}$ was negative control, targeting none of mRNAs.

\section{Cell viability assay}

Non-transfected or transfected cells in 96-well plates were exposed to various concentrations of cisplatin (Meilun, China) for 48 hours, then MTT (Sigma, USA) was added. Cell viability was assessed by the absorbance of each well read by microplate reader (Thermo scientific) at the wavelength of $492 \mathrm{~nm}$.

\section{Quantitative real-time PCR}

Cells were harvested 48 hours post-transfection. TRIzol reagent (Invitrogen) was used to extract the total RNA. The procedure of complementary DNA (cDNA) synthesis and real-time PCR was seen in the previous publication [16]. The sequences of primers (Sangon Biotech, China) were listed in Supplymentary Table S1. U6 was used as internal control.

\section{Dual reporter luciferase assay}

Three 3'-UTR recombinant constructs were generated using dual luciferase plasmid system (a vector containing the Renilla and Firely luciferase gene), including Luc-APAF1-3'-UTR, Luc-PTEN-3'UTR and Luc-PIK3R3-3'-UTR, each of which including the potential biding sequences to miR-186. The Luc-3'-UTR vector and miR-186 mimic or NC were co-transfected into HEK 293T cells, 24 hours later, luciferase activity was tested [16].

\section{Western blotting}

Proteins were extracted from cells 48 hours post-transfection with miR-186 mimic or NC. Antibodies to PTEN (Multi Sciences, Hangzhou, China), PIK3R3 (Multi Sciences), APAF1 (Bioss, Bejing, China) or GAPDH (Multi Sciences) were used. The procedure was seen in the previous publication [16]. GAPDH was used as internal control. 


\section{Statistical analysis}

All experiments were conducted at least three independent performances, and data were shown as the mean \pm standard deviation. Differences between two samples were analyzed by the two-tailed Student's t-test. Statistical significance was accepted at $P<0.05$.

\section{Results}

\section{Downregulation of miR-186 in cisplatin-resistant ovarian cells}

We confirmed the cisplatin resistance of SKOV3/DDP and A2780/DDP cell lines conserved in our lab. Cells in 96-well plates were treated with different concentrations of cisplatin, 48 hours later, the $50 \%$ inhibitory concentration (IC50) was assessed by cell viability assay. The IC50 of cisplatin was about two-fold in SKOV3/DDP and A2780/DDP cells than their parental SKOV3 and A2780 cells, respectively (Figure 1A, B), it revealed SKOV3/DDP and A2780/ DDP cells were indeed cisplatin-resistant ovarian cancer cells. To verify the alterations of miR-186 in cisplatin-resistant ovarian cells, total RNA was extracted from cisplatin-resistant ovarian cancer cells and their parental cells. The results of quantitative real-time PCR showed that the levels of miR-186 were markedly downregulated in cisplatin-resistant ovarian cancer cells SKOV3/DDP and A2780/DDP, in comparison with their corresponding parental cells (Figure 1C, D). Our results were concordant to the previous reports $[14,15]$.

\section{Bidirectional regulatory effects of miR-186 on cisplatin sensitivity of ovarian cancer cells}

To study the role of miR-186 in regulation of cisplatin sensitivity, different concentrations of miR-186 mimic or NC were transfected into cisplatin-resistant ovarian cancer cells A2780/DDP. Total RNA was extracted 48 hours after transfection, and the transfection efficiency was evaluated by the expression levels of miR-186. The results of quantitative real-time PCR exhibited the levels of miR-186 were effectively increased by transfection of miR-186 mimic at $20 \mathrm{nM}, 40 \mathrm{nM}, 80 \mathrm{nM}$, respectively (Figure 2A). The IC50 of cisplatin was assessed by cell viability assay. In comparison with the NC group, we found that the IC50 was downregulated in cells transfected with miR-186 mimic at $20 \mathrm{nM}$, while the IC50 was upregulated when transfected with miR-186 mimic at $80 \mathrm{nM}$ and no significant change when transfected with miR-186 mimic at 40nM (Figure 2B). The results revealed that the treatment with miR-186 mimics at low concentration $(20 \mathrm{nM})$ increased cisplatin sensitivity of
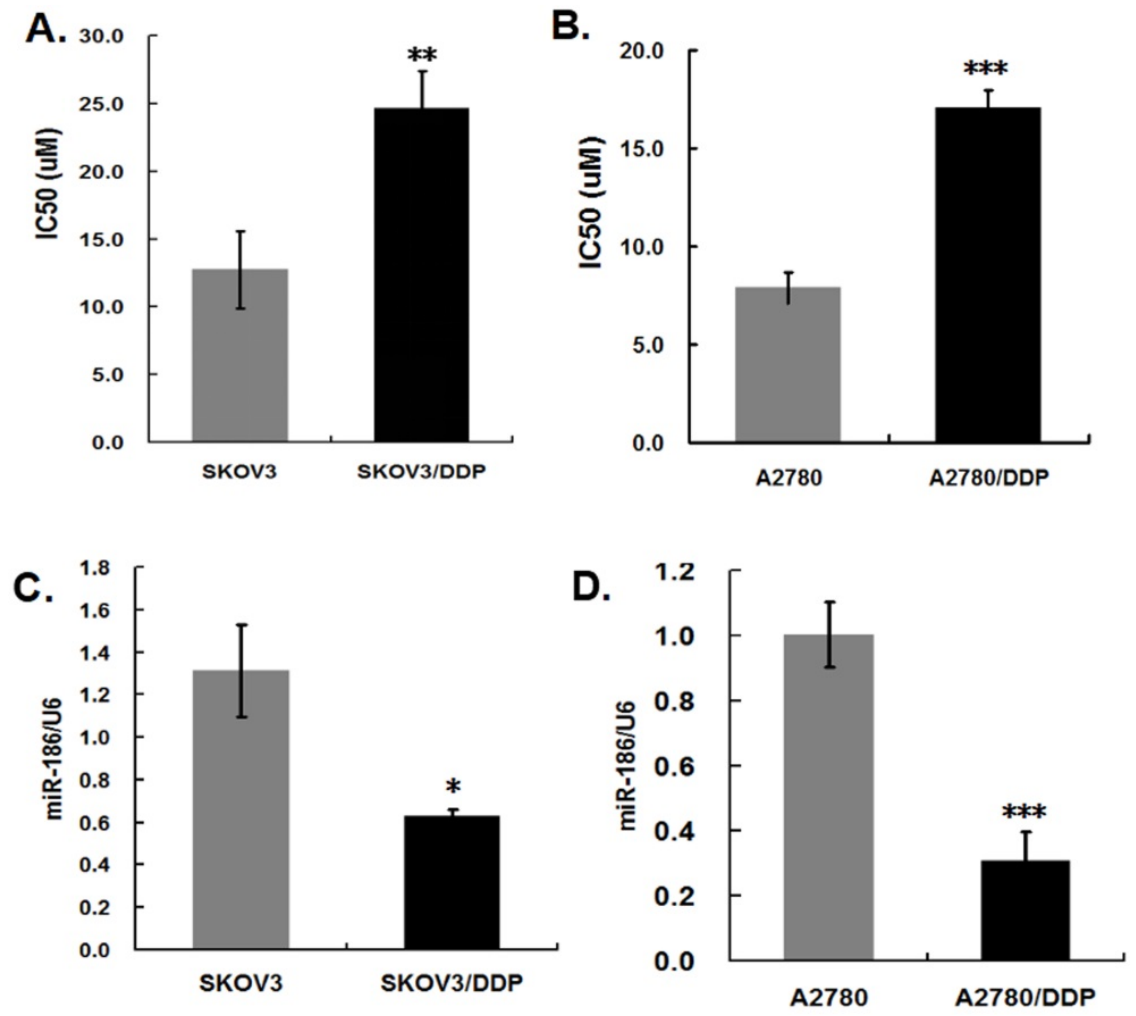

Figure 1. The expression levels of miR-186 in cisplatin-resistant and the parental ovarian cancer cells. (A) (B) Cells were treated with cisplatin for 48 hours, and then the IC50 of cisplatin was evaluated by cell viability assay. (C) (D) The expression levels of miR-186 was analyzed by quantitative real-time PCR in cisplatin-resistant and the parental ovarian cancer cells. $* P<0.05, * * P<0.01, * * * P<0.001$. A2780/DDP cells but decreased cisplatin sensitivity at high concentration $(80 \mathrm{nM})$. It indicated miR-186 bidirectionally regulated cisplatin sensitivity of ovarian cancer cells in a dose-dependent manner.

\section{Bidirectional regulatory effects of miR-186 was dependent on cell types}

To further investigate whether miR-186 play bidirectional regulatory effects on cisplatin sensitivity in other types cancer cell lines, SKBR3, HepG2, A549 and HCT116 cells were transfected with various concentrations of miR-186 mimic or $\mathrm{NC}$, and then treated with or without cisplatin at 20uM. Cell viability assay showed transfection of miR-186 at the concentration of 20nM markedly inhibited cell survival in all of these cells compared with NC group, it revealed miR-186 mimic at the concentration of $20 \mathrm{nM}$, increased 
cisplatin sensitivity in all of these cells. Obviously, miR-186 higher than 40nM lost its inhibitory effects in SKBR3, and miR-186 at 80nM lost its inhibitory effects in HepG2 cells. In A549 and HCT116 cells, miR-186 was effective in inhibiting cell viability, increasing cisplatin sensitivity even the concentration was as high as $80 \mathrm{nM}$ (Figure 3). These results revealed that the role of miR-186 in increasing cisplatin sensitivity was dependent on its dose and cell types. This may be due to various abundance of miR-186 and its targets in different types of cancer cells.

\section{The multiple opposite functional targets may contribute to the bidirectional regulatory effects of miR-186}

In order to explore the mechanism of the bidirectional regulatory effects of miR-186 on cisplatin sensitivity of ovarian cancer cells, a series of bioinformatic softwares (including PITA, TargetScan, PicTar, microT, miRanda) were used to analyze the potential targets of miR-186 as far as possible. PIK3R3, PTEN and APAF1 were predicted to be potential targets of miR-186 (Figure 4A). PIK3R3, an important regulatory subunit of PI3K, promotes AKT activated. PTEN, a negative regulatory molecule of AKT pathway, is opposed to PI3K activity. Activated AKT is able to promote cell survival, proliferation. APAF1, an important molecule which combines with cytochrome c and Caspase9 precursor, leads to Caspase9 activation, thus promotes apoptosis [17]. The dual-reporter luciferase assay showed miR-186 suppressed the 3'-UTR of PTEN, PIK3R3 and APAF1 directly (Figure 4B).

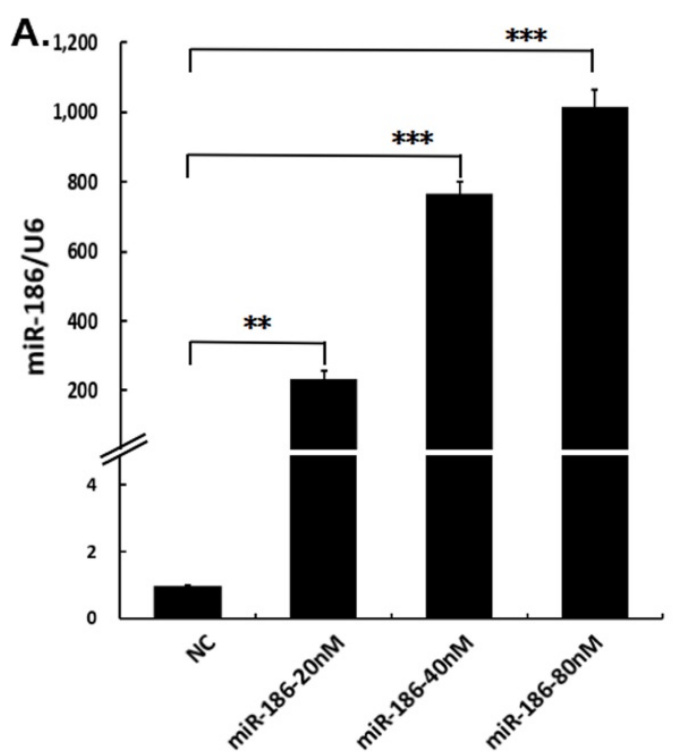

Overexpression of miR-186 significantly reduced the protein levels of PTEN and PIK3R3 in a dosedependent manner compared with the NC group in A2780/DDP cells (Figure 4C, D). We concluded that PTEN and PIK3R3 were two targets in cisplatinresistant ovarian cancer cells. However, miR-186 significantly increased the protein levels of APAF1 in a dose-dependent manner (Figure 4C, D). Moreover, we analyzed the co-expression of miR-186 and its targets in cancer tissues, and the data (collected from ENCORI database) showed an inverse correlation between miR-186 and PTEN, miR-186 and PIK3R3 in multiple cancer types, including in non-small-cell lung cancer, colon cancer, hepatic carcinoma, and breast cancer, and a positive correlation between miR-186 and APAF1 in several cancer types (Table 1). In addition, Dong et al. observed significant inverse correlation $(\mathrm{r}=-0.524, P<0.0001)$ between miR-186 and PIK3R3 in 200 EOC tissues [18]. It validated that miR-186 could suppress the expression PTEN and PIK3R3, and enhance the expression APAF1.

Based on the above, we proposed the final effects of PTEN and APAF1 outweighed PIK3R3 when miR-186 at low concentration so as to increase the cisplatin sensitivity of ovarian cancer cells, while the final effects of PIK3R3 outweighed PTEN and APAF1 when miR-186 at high concentration so as to decrease the cisplatin sensitivity. We concluded the outcome of these opposite functional molecules contributed to the bidirectional regulatory effects of miR-186 on cisplatin sensitivity of ovarian cancer cells (Figure 5).

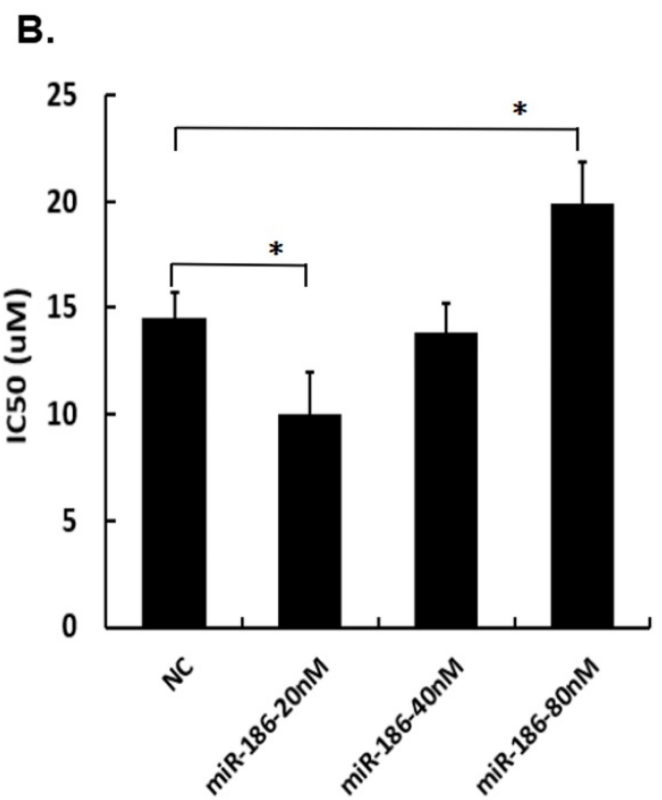

Figure 2. The bidirectional regulatory effects of miR-186 on cisplatin sensitivity of ovarian cancer cells. (A) A2780/DDP cells were transfected with different concentrations of miR-186 mimic or NC, 48 hours later, cells were collected and the levels of miR-186 were assessed by quantitative real-time PCR. (B) Transfected A2780/DDP cells with different concentrations of miR-186 mimic or NC, were treated with various concentrations of cisplatin for a further 48 hours, then the IC 50 was evaluated by cell viability. $* P<0.05, * * P<0.01, * * * P<0.001$ 


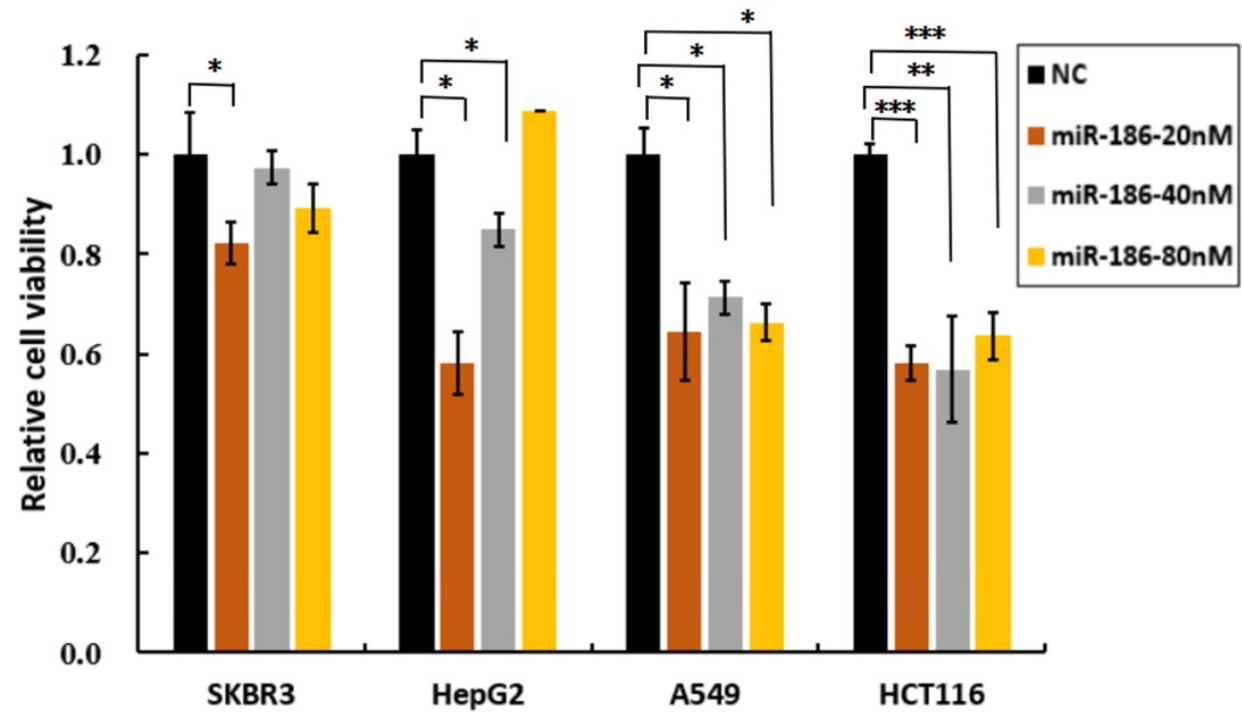

Figure 3. The dose-dependent effects of miR-186 on cisplatin sensitivity in other types of cancer cells. Transfected cells (including SKBR3, HepG2, A549 and HCT116 cells) with different concentrations of miR-186 mimic or NC, were exposed to cisplatin at the concentration of $20 \mathrm{uM}$, and cell viability was analyzed 48 hours later. $* P<0.05, * * P<0.01, * * * P<0.001$.

A.

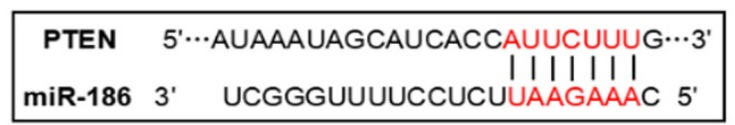

PIK3R3 5'*GACUACGAUGAGGGCAUUCUUUC $\cdots 3^{\prime}$ $\begin{array}{lll}\text { miR-186 } & \text { 3' I I I I I I I } \\ \end{array}$

$\begin{array}{lll}\text { APAF1 } & 5 ' \cdots \text { GGCAUUGUGAAUGCCAUUCUUUU } \cdots 3^{\prime} \\ \text { miR-186 } & 3^{\prime} \text { UCGGGUUUUCCUCUUAAGAAAC } & 5\end{array}$

C.
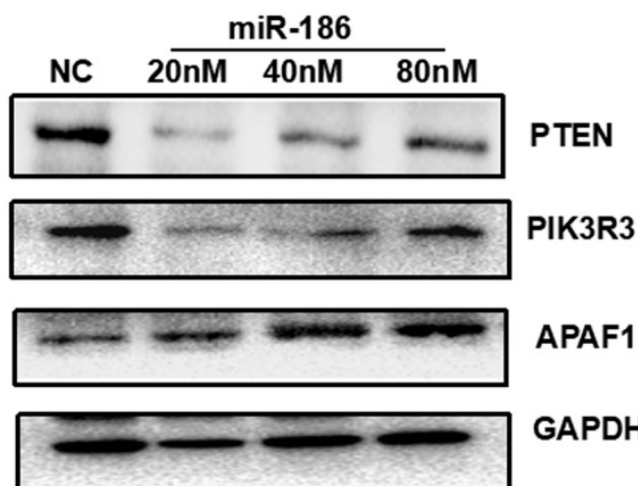

APAF1

\section{GAPDH}

B.

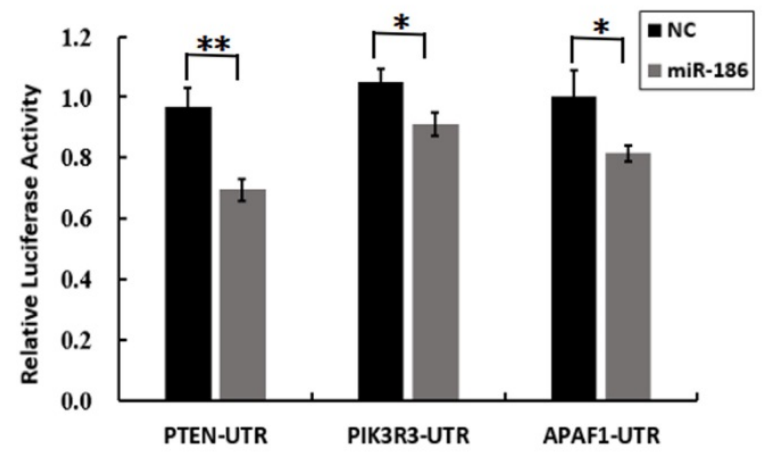

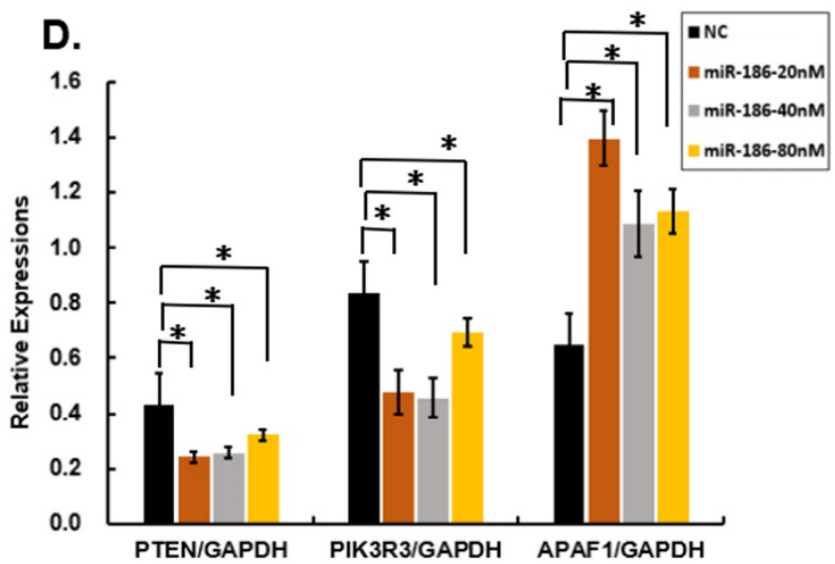

Figure 4. Multiple targets of miR-186. (A) The predicated targets PIK3R3, PTEN and APAF1 were showed in bioinformatics database. (B) HEK 293T cells were co-transfected with miR-186mimic or NC and a luciferase recombinant with the 3'-UTR of PIK3R3, or APAF1, or PTEN, then the relative luciferase activity was detected 24 hours later. (C) The protein levels of PIK3R3, APAF1 and PTEN were measured by western blotting 48 hours after transfection with miR-186 mimic or NC in A2780/DDP cells. (D) The quantitative analysis of protein levels (normalized to GAPDH) by Image J. $* P<0.05$.

\section{Discussion}

MiR-186, generated from the intron 8 of its host gene ZRANB2, was parallelly transcribed with its host gene [19]. Majority of studies showed miR-186 served as a tumor repressor miRNA in various malignancies such as carcinomas of prostate [9], breast [10] and liver [11], which repressed proliferation and migration, and promoted apoptosis. Some studies verified that miR-186 served as an oncomir in endometrial cancer and squamous cell carcinoma, which promoted proliferation and migration, and inhibited apoptosis $[12,13]$. 
Table 1. The Co-Expression Analysis for miR-186 and targets (from ENCORI database)

\begin{tabular}{|c|c|c|c|c|}
\hline Correlation & Cancer Types & Sample Num. & $\begin{array}{l}\text { Coefficient } \\
-r\end{array}$ & p-value \\
\hline \multirow[t]{14}{*}{ miR-186 VS PTEN } & Breast Invasive Carcinoma & 1085 & -0.172 & 1.17E-08 \\
\hline & Colon Adenocarcinoma & 450 & -0.112 & $1.80 \mathrm{E}-02$ \\
\hline & Liver Hepatocellular Carcinoma & 370 & -0.11 & $3.42 \mathrm{E}-02$ \\
\hline & Lung Adenocarcinoma & 512 & -0.161 & 2.64E-04 \\
\hline & Lung Squamous Cell Carcinoma & 475 & -0.096 & $3.68 \mathrm{E}-02$ \\
\hline & Head and Neck Squamous Cell Carcinoma & 497 & -0.103 & $2.15 \mathrm{E}-02$ \\
\hline & Kidney Renal Papillary Cell Carcinoma & 289 & -0.300 & $1.98 \mathrm{E}-07$ \\
\hline & Bladder Urothelial Carcinoma & 408 & -0.227 & $3.71 \mathrm{E}-06$ \\
\hline & Acute Myeloid Leukemia & 83 & -0.308 & 4.67E-03 \\
\hline & Brain Lower Grade Glioma & 525 & -0.093 & $3.36 \mathrm{E}-02$ \\
\hline & Pheochromocytoma and Paraganglioma & 183 & -0.308 & $2.26 \mathrm{E}-05$ \\
\hline & Rectum Adenocarcinoma & 161 & -0.302 & $9.82 \mathrm{E}-05$ \\
\hline & Stomach Adenocarcinoma & 372 & -0.256 & $5.62 \mathrm{E}-07$ \\
\hline & Uterine Corpus Endometrial Carcinoma & 538 & -0.145 & 7.17E-04 \\
\hline \multirow[t]{3}{*}{ miR-186 VS PIK3R3 } & Testicular Germ Cell Tumors & 156 & -0.318 & $5.35 \mathrm{E}-05$ \\
\hline & Thymoma & 119 & -0.305 & 7.31E-04 \\
\hline & Kidney Renal Clear Cell Carcinoma & 517 & -0.092 & $3.70 \mathrm{E}-02$ \\
\hline \multirow[t]{5}{*}{ miR-186 VS APAF1 } & Liver Hepatocellular Carcinoma & 370 & 0.146 & $4.84 \mathrm{E}-03$ \\
\hline & Esophageal Carcinoma & 162 & 0.189 & $1.61 \mathrm{E}-02$ \\
\hline & Kidney Renal Clear Cell Carcinoma & 517 & 0.096 & $2.96 \mathrm{E}-02$ \\
\hline & Skin Cutaneous Melanoma & 449 & 0.242 & $2.10 \mathrm{E}-07$ \\
\hline & Stomach Adenocarcinoma & 372 & 0.111 & $3.17 \mathrm{E}-02$ \\
\hline
\end{tabular}

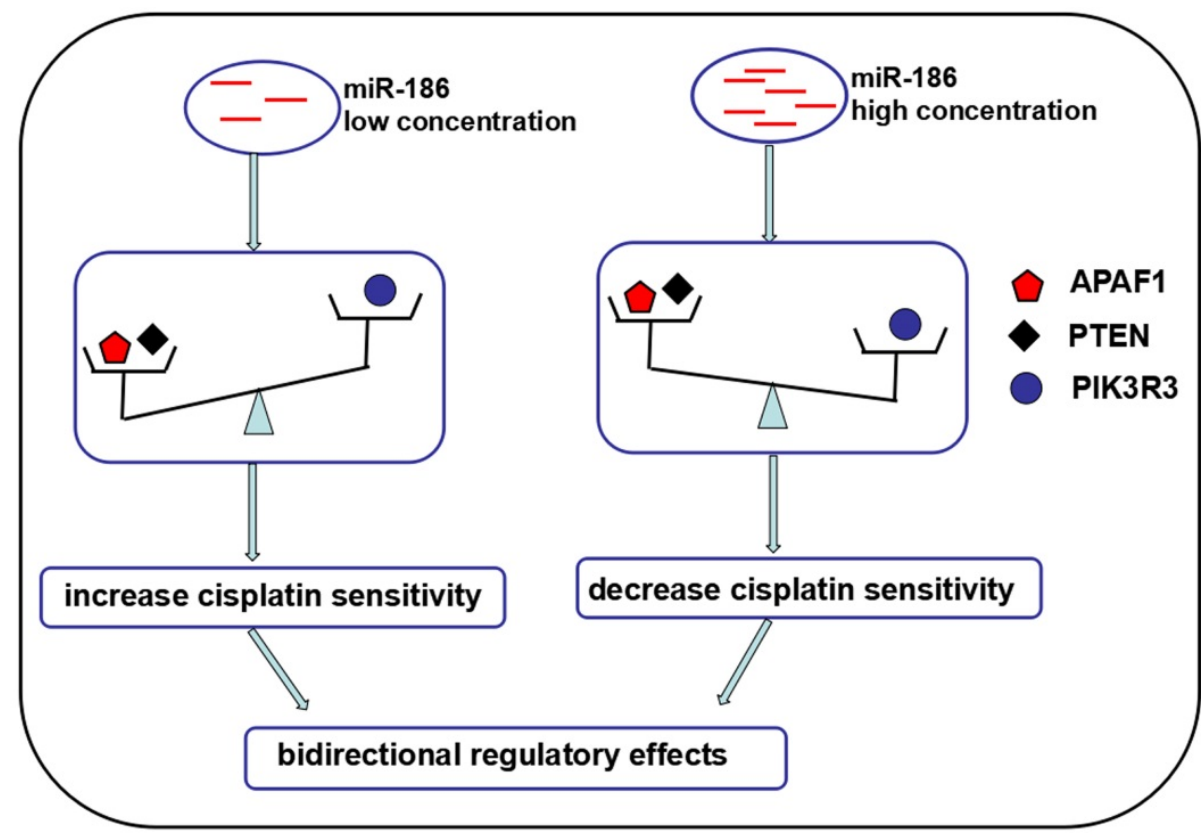

Figure 5. The general view of multiple targets or regulated molecules contributing to the bidirectional regulatory effects of miR-186 on cisplatin sensitivity. The final effects of PTEN and APAF1 outweighed PIK3R3 when miR-186 at low concentration so as to increase the cisplatin sensitivity of ovarian cancer cells, while the final effects of PIK3R3 outweighed PTEN and APAF1 when miR-186 at high concentration so as to decrease the cisplatin sensitivity.

Zhu et al. collected series of ovarian cancer samples from patients with FIGO stage IIIC or IV $(n=52)$, who were treated with the standard care of platinum-based therapy after surgery, and found miR-186 was greatly reduced in tumor specimens from patients with PFS (progression-free survival) $<6$ months (platinum resistant), compared with PFS $>6$ months (platinum sensitive) [15]. MiR-186 was also downregulated in the cisplatin-resistant ovarian cell lines and ectopic overexpression of miR-186 increased cisplatin sensitivity in vitro [14, 15]. However, we found the bidirectional regulatory effects of miR-186 on cisplatin sensitivity for the first time that overexpression of miR-186 at low concentration increased the cisplatin sensitivity of ovarian cancer cells A2780/DDP, while high concentration of miR-186 decreased the cisplatin sensitivity. Moreover, the bidirectional regulatory effects of miR-186 was also dependent on cell types (Figures 2, 3). The similarly bidirectional effects of another miRNA was 
also reported: miR-181a inhibited the cell viability of breast cancer cells significantly when miR-181a dose lower than $50 \mathrm{nM}$, but promoted proliferation rather than inhibitory effect when miR-181a dose higher than 50nM [20]. We concluded that miR-186 played bidirectional regulatory effects on cisplatin sensitivity in dose and cell type dependent manners.

We suspected multiple targets of miR-186 affecting pathways simultaneously contributed to its bidirectional regulatory effects. It was demonstrated that PTEN and PIK3R3 were two functional targets of miR-186 in lung adenocarcinoma [21] and in ovarian cancer [18]. However, the abundance of predicated targets varies in different cell types [22]. It is still necessary to verify whether they are functional targets of miR-186 in regulation of cisplatin sensitivity of ovarian cancer cells. We verified PIK3R3 and PTEN were the two targets of miR-186, and overexpression of miR-186 decreased the protein levels of PIK3R3 and PTEN in cisplatin-resistant ovarian cancer cells (Figures 4).

PTEN, a negative regulator of the PI3K/AKT signaling pathway, was an important molecule in regulating cisplatin sensitivity [23]. Fu et al. showed both the mRNA and protein levels of PTEN was decreased in CDDP-resistant ovarian cancer tissues $(\mathrm{N}=5)$ compared with CDDP-sensitive ovarian cancer tissues $(\mathrm{N}=5)$ [24]. Here were reports that PTEN was a direct target of miR-214 and miR-93 which induced cisplatin resistance in ovarian cancer [24, 25]. That was to say miR-186 may decrease cisplatin sensitivity via suppressing PTEN. PIK3R3, one of the regulatory subunits of PI3K, could activate AKT pathway. In ovarian cancer, Zhang revealed PIK3R3 was upregulated significantly in cancer samples $(\mathrm{N}=28)$ compared with normal ovary $(\mathrm{N}=4)$ [26]. Knockdown or silence of PIK3R3 decreased cell proliferation, migration and invasion, and increased apoptosis [27]. Therefore, miR-186 may increase cisplatin sensitivity via suppressing PIK3R3.

APAF1, an important molecule promoting apoptosis [17], was downregulated in series of ovarian carcinoma samples with lymph node metastasis, and at the advanced FIGO stage [28]. APAF1 was a validated target of miR-186 in cutaneous squamous cell carcinoma [12]. In this study, the dual-reporter luciferase assay showed miR-186 suppressed the 3'-UTR of APAF1. However, overexpression of miR-186 significantly increased the protein levels of APAF1 in comparison with the NC group in A2780/DDP cells (Figures 4). We considered transfection of miR-186 mimic in A2780/DDP cells induced changes of targets pools or miRNA pools that lead to the upregulation of APAF1. Downregulation of APAF1 expression by miR-155 decreased the cisplatin sensitivity of A549 cells [29]. Otherwise, upregulation of APAF1 gene expression contributed to miR-186 in increasing cisplatin sensitivity of ovarian cancer cells.

In conclusion, we verified that miR-186 was downregulated in cisplatin-resistant ovarian cancer cells, low concentration of miR-186 increased cisplatin sensitivity of ovarian cancer cells, while high concentration of miR-186 displayed the opposite function. The bidirectional regulatory effects of miR-186 was dependent on its dose and cell types. Further study revealed that miR-186 suppressed PTEN and PIK3R3 expression by targeting 3'UTRs directly, but increased the protein levels of APAF1. MiR-186 may increase cisplatin sensitivity by suppressing PIK3R3 and upregulation of APAF1, also may decrease cisplatin sensitivity by suppressing PTEN. We proposed the final effects of PTEN and APAF1 outweighed PIK3R3 when miR-186 at low concentration so as to increase the cisplatin sensitivity of ovarian cancer cells, while the final effects of PIK3R3 outweighed PTEN and APAF1 when miR-186 at high concentration so as to decrease the cisplatin sensitivity (Figure 5). We concluded the outcome of these opposite functional molecules contributed to the bidirectional regulatory effects of miR-186 in ovarian cancer cisplatin sensitivity.

\section{Abbreviations}

PTEN: phosphatase and tensin homolog; PIK3R3: phosphoinositide 3-kinase regulatory subunit 3; APAF1: apoptotic protease activating factor-1.

\section{Supplementary Material}

Supplementary table S1.

http://www.jcancer.org/v11p3446s1.pdf

\section{Acknowledgement}

This work was supported by the National Natural Science Foundation of China (81602303).

\section{Competing Interests}

The authors have declared that no competing interest exists.

\section{References}

1. Bray F, Ferlay J, Soerjomataram I , et al. Global cancer statistics 2018: GLOBOCAN estimates of incidence and mortality worldwide for 36 cancers in 185 countries. CA Cancer J Clin 2018; 68(6):394-424.

2. Barabas K, Milner R, Lurie D, et al. Cisplatin: a review of toxicities and therapeutic applications. Vet Comp Oncol 2008; 6(1):1-18.

3. Friedlander $\mathrm{M}$, Trimble E, Tinker A et al. Clinical trials in recurrent ovarian cancer. Int J Gynecol Cancer 2011; 21(4):771-775.

4. Li H, Zeng J, Shen K. PI3K/AKT/mTOR signaling pathway as a therapeutic target for ovarian cancer. Arch Gynecol Obstet 2014; 290(6):1067-1078.

5. Gohr K, Hamacher A, Engelke LH, et al. Inhibition of PI3K/Akt/mTOR overcomes cisplatin resistance in the triple negative breast cancer cell line HCC38. BMC Cancer 2017; 17(1):711. 
6. Zhao Z, Wang J, Tang J, et al. JNK- and Akt-mediated Puma expression in the apoptosis of cisplatin-resistant ovarian cancer cells. Biochem J 2012; 444(2):291-301.

7. Kwon JJ, Factora TD, Dey S, et al. A Systematic Review of miR-29 in Cancer. Mol Ther Oncolytics 2019; 12:173-194.

8. Shi X, Xiao L, Mao X, et al. miR-205-5p Mediated Downregulation of PTEN Contributes to Cisplatin Resistance in C13K Human Ovarian Cancer Cells. Front Genet 2018; 9:555.

9. Lu S, Wang MS, Chen PJ, et al. miRNA-186 inhibits prostate cancer cell proliferation and tumor growth by targeting YY1 and CDK6. Exp Ther Med 2017; 13(6):3309-3314.

10. Sun WJ, Zhang YN, Xue P. miR-186 inhibits proliferation, migration, and epithelial-mesenchymal transition in breast cancer cells by targeting Twist1. J Cell Biochem 2019; 120(6):10001-10009.

11. Ruan T, He X, Yu J, et al. MicroRNA-186 targets Yes-associated protein 1 to inhibit Hippo signaling and tumorigenesis in hepatocellular carcinoma. Oncol Lett 2016; 11(4):2941-2945.

12. Tian J, Shen $R$, Yan $Y$, et al. miR-186 promotes tumor growth in cutaneous squamous cell carcinoma by inhibiting apoptotic protease activating factor-1. Exp Ther Med 2018; 16(5):4010-4018.

13. Myatt SS, Wang J, Monteiro LJ , et al. Definition of microRNAs that repress expression of the tumor suppressor gene FOXO1 in endometrial cancer. Cancer Res 2010; 70(1):367-377.

14. Sun KX, Jiao JW, Chen S, et al. MicroRNA-186 induces sensitivity of ovarian cancer cells to paclitaxel and cisplatin by targeting ABCB1. J Ovarian Res 2015; $8: 80$.

15. Zhu $X$, Shen $H$, Yin $X$, et al. miR-186 regulation of Twist1 and ovarian cancer sensitivity to cisplatin. Oncogene 2016; 35(3):323-332.

16. Xiang Y, Ma N, Wang D, et al. MiR-152 and miR-185 co-contribute to ovarian cancer cells cisplatin sensitivity by targeting DNMT1 directly: a novel epigenetic therapy independent of decitabine. Oncogene 2014; 33(3):378-386.

17. $\mathrm{Hu} \mathrm{Q}, \mathrm{Wu} \mathrm{D}, \mathrm{Chen} \mathrm{W}$, et al. Molecular determinants of caspase- 9 activation by the Apaf-1 apoptosome. Proc Natl Acad Sci U S A 2014; 111(46):16254-16261.

18. Dong $\mathrm{S}$, Wang $\mathrm{R}$, Wang $\mathrm{H}$, et al. HOXD-AS1 promotes the epithelial to mesenchymal transition of ovarian cancer cells by regulating miR-186-5 $p$ and PIK3R3. J Exp Clin Cancer Res 2019; 38(1):110.

19. Antoniou A, Mastroyiannopoulos NP, Uney JB , et al. miR-186 inhibits muscle cell differentiation through myogenin regulation. J Biol Chem 2014; 289(7):3923-3935.

20. Yang C, Tabatabaei SN, Ruan X, et al. The Dual Regulatory Role of MiR-181a in Breast Cancer. Cell Physiol Biochem 2017; 44(3):843-856.

21. Feng $\mathrm{H}$, Zhang $\mathrm{Z}$, Qing X, et al. miR-186-5p promotes cell growth, migration and invasion of lung adenocarcinoma by targeting PTEN. Exp Mol Pathol 2019; 108:105-113.

22. Hendrickson DG, Hogan DJ, McCullough HL, et al. Concordant regulation of translation and mRNA abundance for hundreds of targets of a human microRNA. PLoS Biol 2009; 7(11):e1000238.

23. Patch AM, Christie EL, Etemadmoghadam D, et al. Whole-genome characterization of chemoresistant ovarian cancer. Nature 2015; 521(7553):489-494

24. Fu X, Tian J, Zhang L, et al. Involvement of microRNA-93, a new regulator of PTEN/Akt signaling pathway, in regulation of chemotherapeutic drug cisplatin chemosensitivity in ovarian cancer cells. FEBS Lett 2012; 586(9):1279-1286.

25. Yang $\mathrm{H}$, Kong $\mathrm{W}, \mathrm{He} \mathrm{L}$, et al. MicroRNA expression profiling in human ovarian cancer: miR-214 induces cell survival and cisplatin resistance by targeting PTEN. Cancer Res 2008; 68(2):425-433.

26. Zhang $\mathrm{L}$, Huang $\mathrm{J}$, Yang $\mathrm{N}$, et al. Integrative genomic analysis of phosphatidylinositol 3'-kinase family identifies PIK3R3 as a potential therapeutic target in epithelial ovarian cancer. Clin Cancer Res 2007; 13(18 Pt 1):5314-5321.

27. Dong L, Hui L. HOTAIR Promotes Proliferation, Migration, and Invasion of Ovarian Cancer SKOV3 Cells Through Regulating PIK3R3. Med Sci Monit 2016; 22:325-331.

28. Abdelrahman AE, Fathy A, Elsebai EA , et al. Prognostic impact of Apaf-1, Cyclin D1, and AQP-5 in serous ovarian carcinoma treated with the first-line chemotherapy. Ann Diagn Pathol 2018; 35:27-37.

29. Zang YS, Zhong YF, Fang Z , et al. MiR-155 inhibits the sensitivity of lung cancer cells to cisplatin via negative regulation of Apaf-1 expression. Cancer Gene Ther 2012; 19(11):773-778. 